\title{
The Third Polarization of Light
}

\author{
Richard A. Hutchin \\ Optical Physics Company, Calabasas, CA, USA \\ Email: rahutchin@opci.com
}

Received 6 February 2015; accepted 24 February 2015; published 27 February 2015

Copyright (C) 2015 by author and Scientific Research Publishing Inc.

This work is licensed under the Creative Commons Attribution International License (CC BY).

http://creativecommons.org/licenses/by/4.0/

(c) (i) Open Access

\begin{abstract}
We are all taught that there are only two polarizations of light because Maxwell's equations only support two polarizations. This is mathematically true for the electromagnetic fields, but we have learned since the days of Maxwell that the "real" electromagnetic field is not the electromagnetic field tensor $F_{\mu v}$ (composed of Electric and Magnetic field terms) but rather the electromagnetic vector potential $A_{\mu}$. When considered carefully, this requires a third polarization of light with very unusual properties. This third polarization of light does not generate electric or magnetic fields but should be detectable by its impact on supercurrents or quantum interference. It is also unavoidable since it automatically appears under Lorentz transformations to different moving frames.
\end{abstract}

\section{Keywords}

\section{Local Theory of Light, Third Polarization, Vector Potential}

\section{Introduction}

As scientists and engineers, most of us are more familiar with Maxwell's equations than with the underlying vector potential. The vector potential $A_{\mu}$ was first created as a mathematical convenience to solve automatically two of Maxwell's four equations. This was helpful algebraically, but since no one knew how to observe $A_{\mu}$ directly, it was taken purely as a solving technique. With the advent of superconductivity, it became clear that the primary field driving supercurents is the vector potential itself. In the theory of superconductors, the London equation [1] [2] shows the supercurrent proportional to the vector potential—a direct measurement of the vector component of $\mathrm{A}$ in the material.

London Equation:

$$
J_{s}=-\left(e^{2} n_{s} / m c\right) A
$$

Here $e=$ the charge of the electron, $n_{s}=$ the density of superconducting electrons, $m=$ the mass of the electron 
and $c=$ the speed of light.

The impact of this equation sunk in more clearly when the world's most sensitive magnetometer was developed, called a SQUID (Superconducting Quantum Interference Device), shown schematically in Figure 1 [3] [4]. A SQUID goes through a complete signal cycle every magnetic flux quantum $=h / 2 e=2.07 \times 10^{-15}$ webers and is sensitive enough to measure the magnetic fields generated in human brains [5]. A SQUID has a loop of superconducting material through which a magnetic field passes to give a total magnetic flux equal to the magnetic field $B$ times the area of the loop. Amazingly, SQUID's detect a magnetic flux inside the loop even if the magnetic field itself never touches any part of the SQUID circuitry. The only field that interacts with the SQUID circuitry is the vector potential $A$, which produces a circular current according to the London equation above. Since the device shows large effects without any $E$ or $B$ field touching the circuit, we have a convincing demonstration that the vector potential $A$ is a real field. Since $E$ and $B$ come from derivatives of the vector potential, we conclude that the vector potential $A$ is the fundamental field of all electromagnetic phenomena.

Once we have established that the vector potential is the fundamental E\&M field, then one quickly finds a third polarization of light which has no electric or magnetic fields but which will interact with superconductors and quantum interference detectors.

For convenient reference, we here show the derivative relationship between the vector potential $A_{\mu}$, the E\&M Field Tensor $F_{\mu v}$ and then the $E$ and $B$ fields themselves.

$$
F_{\mu v}=A_{\mu, v}-A_{v, \mu}=\left[\begin{array}{cccc}
0 & E_{X} & E_{y} & E_{z} \\
-E_{X} & 0 & B_{z} & -B_{y} \\
-E_{y} & -B_{z} & 0 & B_{X} \\
-E_{z} & B_{y} & B_{X} & 0
\end{array}\right]
$$

\section{The Third Polarization}

With that background we consider the form of a plane wave using the vector potential. The most general form any plane wave can have is given by Equation (3), which shows a plane wave of wave vector $k_{v}$, where all indices $\mu$ and $\tau$ go from 0 to 3 with 0 being time.

$$
A_{\mu}=A 0_{\mu} \mathrm{e}^{i k_{\tau} x^{\tau}}
$$

This most general form of a plane wave is usually then constrained by the Lorentz condition in Equation (4), which ensures that there is no radiating charge density in the E\&M waves.

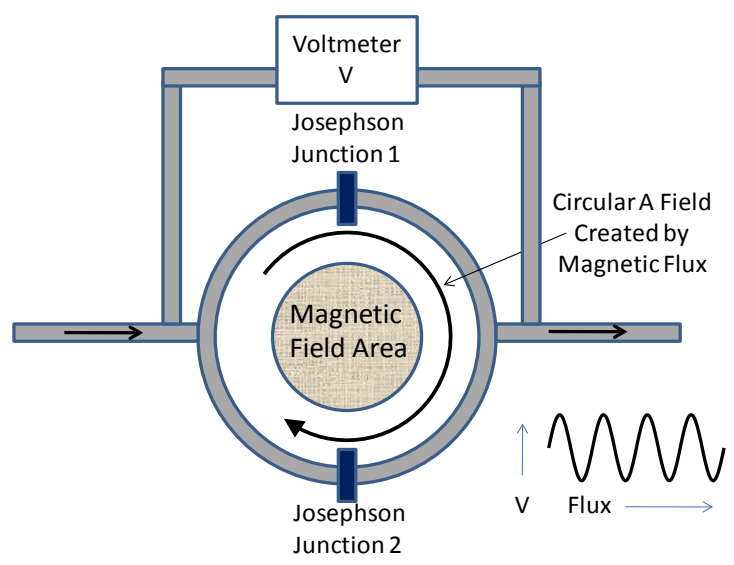

Figure 1. A SQUID (Superconducting Quantum Interference Device) measures the magnetic flux inside the loop using the voltage across a pair of Josephson junctions. The signal goes through one oscillation every magnetic flux quantum $(h / 2 e)$ and is the most sensitive magnet field detector in the world. It measures the magnetic flux even when no magnetic or electric field touches any part of the circuit. 
Lorentz Condition

$$
A_{\mu} k^{\mu}=0
$$

Putting the two together, the following form is often used for a plane wave of $\mathrm{X}$ polarization moving in the $\mathrm{Z}$ direction.

$$
A_{\mu}=\left[\begin{array}{c}
0 \\
A 0_{x} \\
0 \\
0
\end{array}\right] \mathrm{e}^{i k_{\mu} x^{\mu}} \text { where } \quad k_{\mu}=\left[\begin{array}{c}
k_{o} \\
0 \\
0 \\
k_{o}
\end{array}\right]
$$

This is an intuitive and convenient form for the vector potential which satisfies the Lorentz condition, but when we transform it to a different Lorentz frame, it does not maintain its form. For instance if we transform to a coordinate system moving at velocity $-\beta c$ in the $x$ direction, we know that the direction of light propagation will tilt from the $z$ direction toward the $\mathrm{x}$ direction. As the Lorentz transform approaches the speed of light $(\beta \rightarrow$ 1), the direction of propagation will approach the pure $\mathrm{x}$ direction, and the electric vector will be perpendicular along the $\mathrm{z}$ axis (Figure 2).

Equation (6) shows the computation. If the form of the vector potential $A^{\mu}$ stayed constant, we would have almost the entire spatial part of the transformed $A^{\mu}$ vector aligned with the $E$ vector along the $z$ axis as $\beta \rightarrow 1$. However, the Lorentz transformation does quite the opposite; it keeps the $z$ component at zero for all velocities. Thus a standard Lorentz transform takes us immediately to a different form of light.

$$
A^{\mu}=\left[\begin{array}{c}
0 \\
A_{X} \\
0 \\
0
\end{array}\right] A^{\mu}=\left[\begin{array}{cccc}
\gamma & \gamma \beta & 0 & 0 \\
\gamma \beta & \gamma & 0 & 0 \\
0 & 0 & 1 & 0 \\
0 & 0 & 0 & 1
\end{array}\right]\left[\begin{array}{c}
0 \\
A_{X} \\
0 \\
0
\end{array}\right]=\left[\begin{array}{c}
A_{X} \gamma \beta \\
\gamma A_{X} \\
0 \\
0
\end{array}\right]
$$

The New Polarization: The reason this happens while keeping all the proper and familiar transformations in the $E$ and $B$ fields is that there is a new mode in the vector potential which produces only zero $E$ and $B$ fields. When $A_{\mu}$ is a multiple of $k_{\mu}$, then the E\&M tensor $F_{\mu \nu}$ vanishes. This is the form of the new polarization. The familiar $\mathrm{X}$ and $\mathrm{Y}$ polarization states will still exist, of course, simply by projecting out this third state.

\section{New Polarization}

$$
A_{\mu} \sim k_{\mu} \rightarrow F_{\mu v}=0
$$

Note that this mode satisfies the Lorentz condition in Equation (4) simply because for light $k_{\mu} k^{\mu}$ always equals zero. Furthermore, since our standard Lorentz transformations are required for any covariant quantity, such as

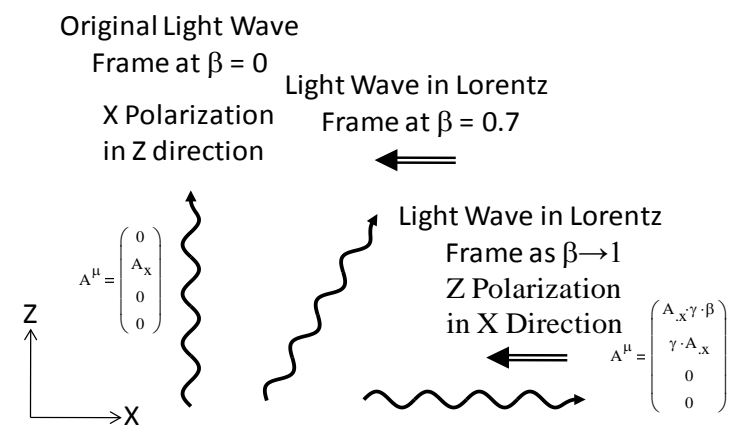

Figure 2. Here we see how a Lorentz transform changes the form of a plane wave. The original plane wave has the simple popular form where the spatial $A$ vector is parallel to the $E$ vector. A Lorentz transform changes that waveform into a completely different form where in the limiting case of a new frame moving close to $-c$ in the $\mathrm{X}$ direction, the spatial part of the $A$ vector is orthogonal to the $E$ vector. 
the vector potential $A_{\mu}$, there appears to be no way to avoid this third polarization state from appearing.

\section{Discussion}

Much of our tradition in E\&M theory is that the electric and magnetic fields are the reality, and the vector potential is a computational convenience. However, for over half a century, the evidence has accumulated that the vector potential is the fundamental field, and the SQUID experiment without any $E$ or $B$ fields touching the circuitry is the extreme verification of that hypothesis. Once we reach that conclusion and show that a third polarization cannot be avoided in a Lorentz invariant universe, then we need to consider the possibility that there is a physical third polarization of light that interacts very differently from the other two.

\section{Conclusions}

Assuming this result of a third polarization stands up to review, it would seem to be an opportunity for experimentalists to try to detect and characterize it. The logical place to begin is an AC superconducting experiment that can differentiate between conventional E\&M waves and vector potential waves. Since the field amplitude matches $\mathrm{k}$, it will generate a supercurrent oscillating in the direction of light propagation, while conventional E\&M light waves create currents perpendicular to the direction of propagation—a simple discriminator.

Also, suppose we take a source of light and pass it through $\mathrm{X}$ and $\mathrm{Y}$ wire grid polarizers. The third polarization, without any $E$ and $B$ fields to generate wire grid currents, would pass through the polarizers and still interact. Alternatively, perhaps this mode will penetrate through room temperature opaque materials without being absorbed well since it has no $E$ and $B$ fields to interact, allowing another detection discriminator.

Experiments always have the last word.

\section{References}

[1] London, F. and London, H. (1935). The Electromagnetic Equations of the Superconductor. Proceedings of the Royal Society A: Mathematical, Physical and Engineering Sciences, 149, 71. http://dx.doi.org/10.1098/rspa.1935.0048

[2] Annett, J.F. (2004) Superconductivity, Superfluids and Condensates. Oxford, 58.

[3] Jaklevic, R.C., Lambe, J., Silver, A.H. and Mercereau, J.E. (1964) Quantum Interference Effects in Josephson Tunneling. Physical Review Letters, 12, 159-160. http://dx.doi.org/10.1103/PhysRevLett.12.159

[4] Kleiner, R., Koelle, D., Ludwig, F. and Clarke, J. (2004) Superconducting Quantum Interference Devices: State of the Art and Applications. Proceedings of the IEEE, 92, 1534. http://dx.doi.org/10.1109/JPROC.2004.833655

[5] Sternickel, K. and Braginski, A.I. (2006) Biomagnetism Using SQUIDs: Status and Perspectives. Superconductor Science and Technology, 19, S160. http://dx.doi.org/10.1088/0953-2048/19/3/024 
Scientific Research Publishing (SCIRP) is one of the largest Open Access journal publishers. It is currently publishing more than 200 open access, online, peer-reviewed journals covering a wide range of academic disciplines. SCIRP serves the worldwide academic communities and contributes to the progress and application of science with its publication.

Other selected journals from SCIRP are listed as below. Submit your manuscript to us via either submit@scirp.org or Online Submission Portal.
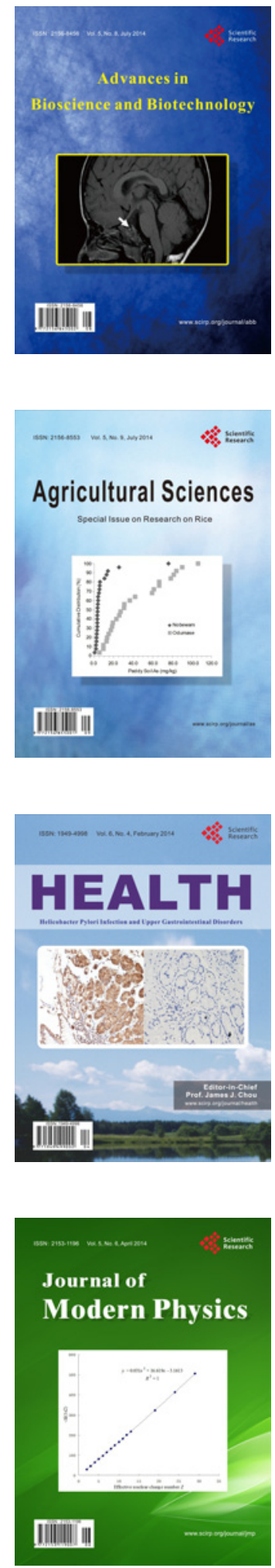
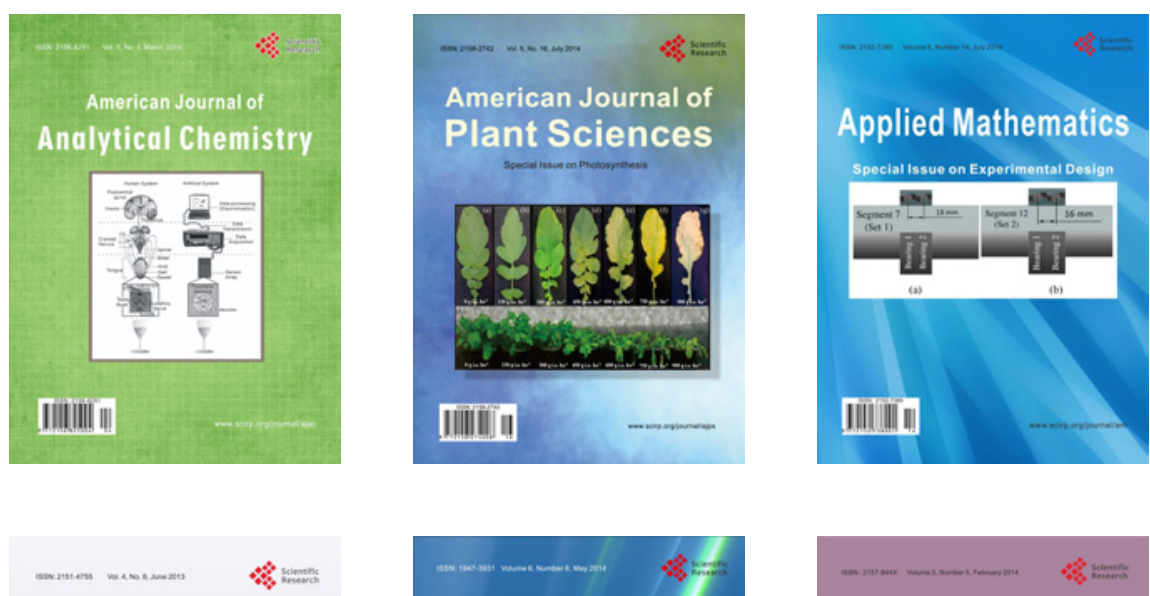

Creative Education
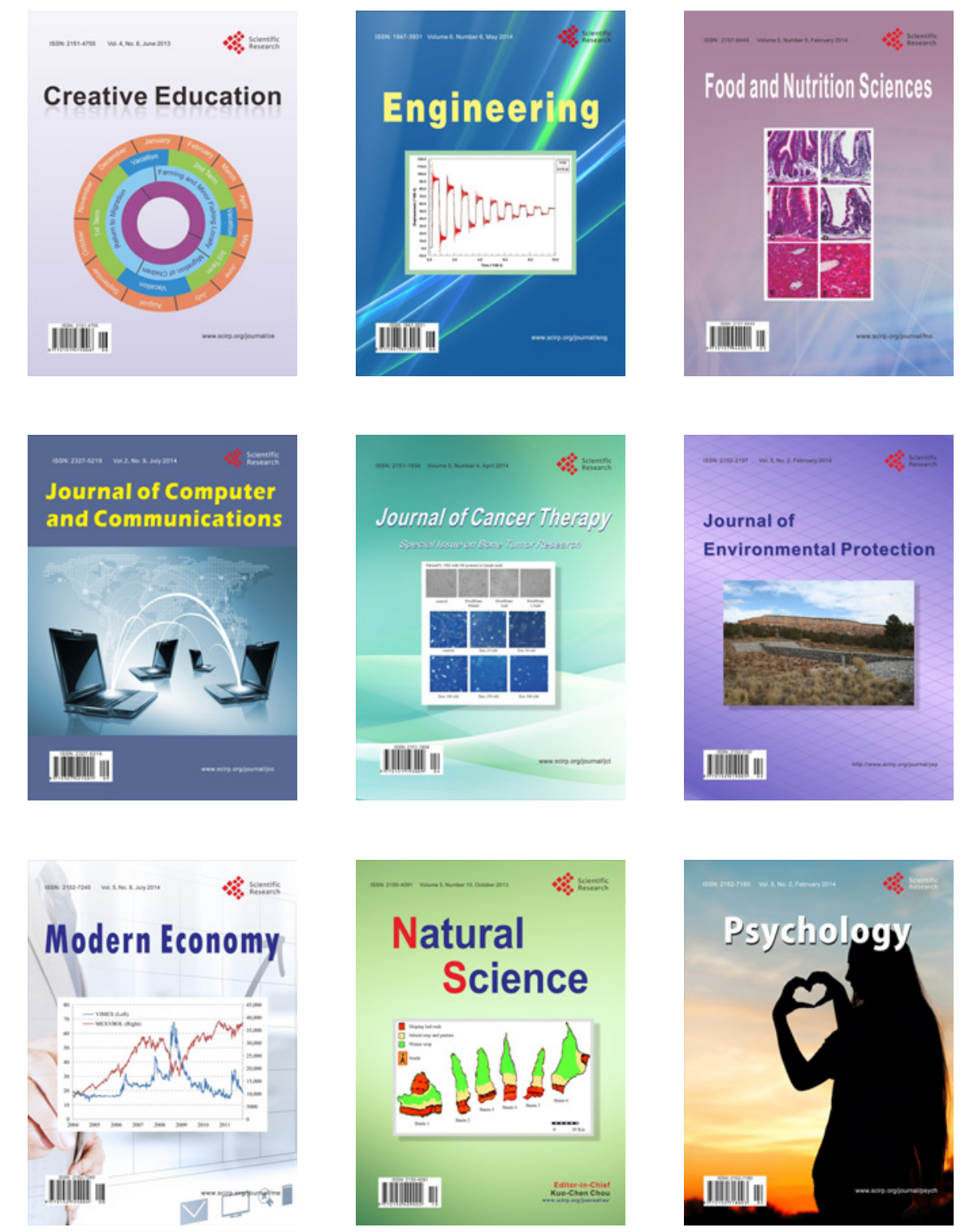